\title{
A Model of Low-Frequency Quasi-Periodic Oscillations in Black Hole X-Ray Binaries
}

\author{
Zhi-Yun Wang ${ }^{1,2}$, Chang-Yin Huang ${ }^{2,3}$, Ding-Xiong Wang ${ }^{2 *}$ and Jiu-Zhou Wang ${ }^{2}$ \\ 1 School of Physics and Electronic Engineering, Xiangfan University, Xiangyang 441053, China; \\ 2 School of Physics, Huazhong University of Science and Technology, Wuhan 430074, China \\ 3 School of Mathematics and Statistics, Huazhong University of Science and Technology, Wuhan 430074, \\ China \\ *dxwang@mail.hust.edu.cn
}

\begin{abstract}
A model of low-frequency quasi-periodic oscillations (LFQPOs) of black hole $\mathrm{X}$-ray binaries (BHXBs) is proposed based on the perturbed magnetohydrodynamic (MHD) equations of accretion disk. It turns out that the LFQPOs frequencies of some BHXBs can be fitted by the frequencies of the toroidal Alfvén wave oscillation corresponding to the maximal radiation flux. In addition, the positive correlation of the LFQPO frequencies with the radiation flux from accretion disk is well interpreted.
\end{abstract}

Key words: accretion,accretion discs - black hole physics - magnetic fields - stars: individual: XTE J1550-564 - stars: individual: GRO J1655-40

\section{INTRODUCTION}

It is well known that the quasi-periodic oscillations (QPOs) have been widely observed in some X-ray binaries, whose compact object is either a neutron star or a black-hole, and QPOs play an essential role as a potentially important tool for studying the strong gravitational field and understanding the physical processes of X-ray states (Done, Gierlinski \& Kubota 2007). Among 20 black hole X-ray binaries (BHXBs), the low-frequency QPOs (LFQPOs) with frequency ranging from a few $m \mathrm{~Hz}$ to $30 \mathrm{~Hz}$ have been detected on one or more occasions for 14 systems in the hard state and the steep power-law (SPL) state (McClintock \& Remillard 2006). Almost all the LFQPOs have some common properties, remaining relatively stable features for days or weeks usually.

A number of theoretical models have been proposed to explain the physical mechanisms of LFQPO. Tagger and Pellat (1999) suggested that LFQPOs observed in low-mass BHXBs can be interpreted by accretion-ejection instability. Titarchuk \& Osherovich (2000) considered that the LFQPOs are caused by the global disk oscillation in the direction normal to the disk, and these oscillations arise from the gravitational interaction between the central compact object and the disk. O'Neill et al. (2011) proposed that LFQPOs are related to the quasi-periodic behavior in global MHD dynamos. Cabanac et al. (2010) presented that an oscillating corona due to magneto-acoustic wave propagating in the corona, and produce multiple QPOs. Kato (2008) proposed that the one-armed c-mode low frequency oscillations of disk are one of possible candidates of LFQPOs based on a resonantly-excited disk-oscillation model. In addition, relativistic precession model was firstly presented by Stella and Vietri (1998), they suggested that LFQPOs are the result of some modulation of Lense-Thirring precession. Later, Schnittman et al. (2006) and Ingram \& Done et al. (2009|2012) developed this model, and successfully explained the variability properties of BHXBs. However, there has been no consensus on the physical nature of LFQPOs.

On the other hand, it has been detected that LFQPO frequencies exhibit a strong positive correlation with disk flux from observations. For example, LFQPO frequencies of XTE J1550-564 (33 observations 
during 1998.9-1999.4), GRO J1655-40 (38 observations during 2005.2.17- 2005.3.6) and H1743-322 (20 observations during 2003.3.28-2003.5.22) display a roughly linear relation with disk flux, when they are in the hard and intermediate states with frequencies in the range $1-7 \mathrm{~Hz}, 0.01-2.5 \mathrm{~Hz}$ and $2-5 \mathrm{~Hz}$, respectively (Remillard \& McClintock 2006; McClintock et al. 2009; Sobczak et al. 2000; Shaposhnikov et al. 2007). Similar quasi-linear relations of LFQPO frequencies with disk flux have been found for GRS 1915+105 (32 observations during 1996.10.29-1998.10.7) in the range 1-15Hz (Markwardt, Swank \& Taam[1999; Sobczak et al.2000; Muno, Remillard \& Morgan 2001) and for XTE J1748-288 (92 observations during 1998.7.131998.9.26) in the range 20-30Hz (Revnivtsev, Trudolyubov \& Borozdin 2000). From the duration time of the above outbursts, we can find that this positive correlation between the LFQPO frequencies and disk flux is on long timescales. It is noted that the QPO observed in XTE J1550-564 during its 1998 outburst shows a correlation between absolute rms amplitude and mean source flux over timescales shorter than $\sim 3 k s$ (Heil,Vaughan \& Uttley 2011), so this relation also holds on short timescales. However, the origin of the correlation between LFQPO frequency and disk flux of BHXBs remains elusive.

If magnetic field is taken into account in accretion disk, a torsional Alfvén wave can be generated by the rotational dragged of space (Koide et al. 2002). We consider a toroidal magnetic field existing in a rotational accretion disk, in which Alfvén wave oscillation propagates along toroidal magnetic field lines due to the perturbation of radial velocity of the accreting matter. Thus the Alfvén wave oscillation will influence the transformation of angular momentum and the radiation flux from the inner disk. Although some researchers suggested that the QPOs of low mass BHXBs arise from Alfvén wave (Zhang 2004; Shi \& $\mathrm{Li}$ 2010; Shi 2011), the association of Alfvén wave oscillation with LFQPOs has not been discussed.

In this paper, we adopt a binary system consisting of a Kerr black hole surrounded by weakly magnetized relativistic thin disk to derive the Alfvén wave frequency based on the perturbed MHD equations, and the LFQPO frequency is fitted by the frequency of Alfven wave propagating in a specific circular orbit of the accretion disk, which corresponds to the maximum radiation flux. In addition, we propose a thin disk model with some corona floating above the disk to well interpret the positive correlation of the LFQPO frequencies with disk flux.

This paper is organized as follows. In section 2, we present a description of our model, and derive the formula for Alfvén wave frequency and radiation flux. In section 3, we fit the LFQPO frequencies of several BHXBs, and fit the positive correlation of LFQPO frequency with the disk flux for two BHXBs, XTE J1550-564 and GRO 1655-40. Finally, in section 4, we discuss the results obtained in our model.

\section{MODEL DESCRIPTION}

\subsection{Alfvén Wave Oscillations in Accretion Disk}

We consider a geometrically thin, optically thick, non-self-gravitating perfect fluid disk, which is magnetized and isothermal, and rotating around a BH. It is assumed that the magnetic pressure is much less than the total pressure $\left(p_{\text {mag }} \ll p\right.$ ), which is a good approximation to a thin Keplerian disk ( $\mathrm{Li}$ 2002). The disk dynamics is governed by the ideal MHD equations given as follows.

$$
\begin{gathered}
\frac{\partial \rho}{\partial t}+\nabla \cdot(\rho \mathbf{v})=0 \\
\frac{\partial \mathbf{v}}{\partial t}+\mathbf{v} \cdot \nabla \mathbf{v}+\frac{1}{\rho} \nabla p+\nabla \Phi+\frac{1}{4 \pi \rho} \mathbf{B} \times(\nabla \times \mathbf{B})=0
\end{gathered}
$$

The magnetic field $\mathbf{B}$ satisfies the induction equation in the MHD approximation, and it reads

$$
\frac{\partial \mathbf{B}}{\partial t}-\nabla \times(\mathbf{v} \times \mathbf{B})=0,
$$

and

$$
\nabla \cdot \mathbf{B}=0 .
$$

The quantities $\rho, \mathbf{v}$ and $p$ in equations (1) and (2) denote mass density, velocity and pressure of plasma, respectively. The Pseudo-Kerr potential (Mukhopadhyay 2002) $\Phi$ is adopted to simulate the effects of 
general relativity, and it reads

$$
\nabla \Phi=-\frac{c^{4}\left(r^{2}-2 a_{*} \sqrt{r}+a_{*}^{2}\right)^{2}}{G M r^{3}\left[\sqrt{r}(r-2)+a_{*}\right]^{2}},
$$

where $r$ is the distance between the plasma to center of BH $\left(r=R / R_{g}\right)$, and $R_{g} \equiv G M / c^{2}$ is the gravitational radius. The quantities $M, G$ and $c$ denote respectively the $\mathrm{BH}$ mass, gravitational constant and speed of light, and $J$ and $a_{*}=J /\left(G M / c^{2}\right)$ represent the BH angular momentum and the dimensionless spin, respectively.

The Keplerian angular velocity in the frame of Pseudo-Kerr potential is derived by Shafee et al. (2008) as follow,

$$
\Omega_{k}=\frac{c^{3}\left(r^{2}-2 a_{*} \sqrt{r}+a_{*}^{2}\right)}{G M r^{2}\left[\sqrt{r}(r-2)+a_{*}\right]} .
$$

The perturbed physical quantities in MHD equations can be written as

$$
p=p_{0}+p^{\prime}, \rho=\rho_{0}+\rho^{\prime}, \mathbf{v}=\mathbf{v}_{0}+\mathbf{v}^{\prime}, \mathbf{B}=\mathbf{B}_{0}+\mathbf{B}^{\prime},
$$

where the subscript ' 0 ' and superscript "' denote the equilibrium and perturbation values, respectively. Assuming that all the perturbations are small enough, i.e., $\rho_{0} \gg \rho^{\prime}, p_{0} \gg p^{\prime}, v_{0} \gg v^{\prime}, B_{0} \gg B^{\prime}$, we can neglect the products of them in second and higher orders. Substituting equations (4) and (7) into equations (1)-(3), we have the linearized MHD perturbation equations as follows,

$$
\begin{gathered}
\left.\frac{\partial \rho^{\prime}}{\partial t}+\rho_{0}\left(\nabla \mathbf{v}^{\prime}\right)+\left(\nabla \rho^{\prime}\right) \cdot \mathbf{v}_{0}\right)=0 \\
\frac{\partial \mathbf{v}^{\prime}}{\partial t}+\mathbf{v}_{\mathbf{0}} \cdot\left(\nabla \mathbf{v}^{\prime}\right)+\frac{c_{s}^{2}}{\rho_{0}} \nabla \rho^{\prime}+\frac{\mathbf{B}_{\mathbf{0}}}{4 \pi \rho_{0}} \times\left(\nabla \times \mathbf{B}^{\prime}\right)=0, \\
\frac{\partial \mathbf{B}^{\prime}}{\partial t}-\nabla \times\left(\mathbf{v}^{\prime} \times \mathbf{B}_{\mathbf{0}}\right)=0
\end{gathered}
$$

From the vertical equilibrium assumption of accretion disk, the half-height $(H)$ of the disk can be written as (Mukhopadhyay 2003),

$$
H=c_{s} R^{1 / 2} F^{-1 / 2}=c_{s} / \Omega_{k},
$$

where $c_{s}=\sqrt{p / \rho}$ is the sound speed.

Incorporating equations (8)-(10), we have the equation for the velocity perturbation as follows,

$$
\frac{\partial^{2} \mathbf{v}^{\prime}}{\partial t^{2}}+\mathbf{v}_{\mathbf{0}} \cdot \nabla\left(\frac{\partial \mathbf{v}^{\prime}}{\partial t}\right)+c_{s}^{2} \nabla\left(\nabla \cdot \mathbf{v}^{\prime}\right)+\mathbf{v}_{\mathbf{A}} \cdot \nabla \times\left[\nabla \times\left(\mathbf{v}^{\prime} \times \mathbf{v}_{\mathbf{A}}\right)\right]=0
$$

where $\mathbf{v}_{\mathbf{A}}=\frac{\mathbf{B}_{\mathbf{0}}}{\sqrt{4 \pi \rho_{0}}}$ is Alfvén velocity, being defined as

$$
v_{A}=\frac{\left|\mathbf{B}_{\mathbf{0}}\right|}{\sqrt{4 \pi \rho_{0}}}=\sqrt{\beta} c_{s}=\sqrt{\beta} H \Omega_{k},
$$

where $\beta=p_{m a g} / p$ is defined as the ratio of magnetic pressure to total pressure.

In order to fit LFQPO frequencies by invoking Alfvén wave propagating in an accretion disk, we express the perturbed velocity as follows,

$$
\mathbf{v}^{\prime}=\mathbf{v}^{\prime} e^{i(\mathbf{k} \cdot \xi-\omega t)},
$$

where $\omega, \xi$ and $\mathbf{k}$ are the perturbation frequency, the displacement vector and the wavenumber vector, respectively. In cylindrical coordinates the wavenumber vector is $\mathbf{k}=k_{r} \hat{\mathbf{r}}+k_{\varphi} \hat{\varphi}+k_{z} \hat{\mathbf{z}}$, where $k_{r}, k_{\varphi}, k_{z}$ represent the radial, azimuthal and vertical components, respectively. Substituting equation (14) into equation (12), we have

$$
-\omega^{2} \mathbf{v}^{\prime}-\omega k \mathbf{v}_{\mathbf{0}} \cdot \mathbf{v}^{\prime}+\left(c_{s}^{2}+v_{A}^{2}\right)\left(\mathbf{k} \cdot \mathbf{v}^{\prime}\right) \mathbf{k}+\left(\mathbf{v}_{\mathbf{A}} \cdot \mathbf{k}\right) \cdot\left[\left(\mathbf{v}_{\mathbf{A}} \cdot \mathbf{k}\right) \mathbf{v}^{\prime}-\left(\mathbf{v}_{\mathbf{A}} \cdot \mathbf{v}^{\prime}\right) \mathbf{k}-\left(\mathbf{k} \cdot \mathbf{v}^{\prime}\right) \mathbf{v}_{\mathbf{A}}\right]=0 .
$$


Considering that Alfvén wave is a transverse wave, transporting along the magnetic field line, we have $\mathbf{k}\left\|\mathbf{B}_{\mathbf{0}}\right\| \mathbf{v}_{\mathbf{0}} \perp \mathbf{v}^{\prime}$, and equation (14) is simplified as

$$
k^{2} v_{A}^{2}-\omega^{2}=0
$$

where the symbols $\|$ and $\perp$ denote parallel and vertical directions, respectively. Thus the angular velocity of the perturbation is related to the Alfvén velocity $v_{A}$ as follows,

$$
\omega=k v_{A}=k \sqrt{\beta} c_{s} .
$$

For a thin magnetized accretion disk, Armitage and Natarajan (1999) presented that the dominant field component is toroidal with saturation occurring when $p_{m a g} \ll p$. So we assume that the unperturbed flow is axisymmetric, and only toroidal magnetic field exists in equilibrium state in the accretion disk,i.e., $\mathbf{B}_{\mathbf{0}}=B_{\varphi} \hat{\varphi}$. Thus a small perturbation of radial velocity of fluid due to accretion of black hole gives rise to the oscillation of the magnetic field $B_{\varphi}$, and results in the Alfvén wave oscillation transporting in the toroidal direction in the disk. For the characteristic wavelength $\lambda \sim R$, the toroidal wave number $k_{\varphi} \sim 2 \pi / R$ (Shi, 2010), and the angular velocity of the perturbation can be written as

$$
\omega=k_{\varphi} \sqrt{\beta} c_{s}=2 \pi \frac{H}{R} \sqrt{\beta} \Omega_{k} .
$$

Inspecting equation (18) we find that $\omega$ is much less than the Keplerian angular velocity $\Omega_{k}$ in the thin disk due to the disk scale height $H$ being much less than disk radius $R$, and this perturbation frequency provides a possibility for fitting LFQPO frequencies of BHXBs.

\subsection{Relation between LFQPO Frequency and Perturbation Frequency}

First of all, we intend to clarify the relation between LFQPO frequency and the perturbation frequency. The disk angular velocity can be regarded as the Keplerian angular velocity, i.e., $\Omega=\Omega_{k}$, provided that the radial magnetic force can be neglected. This result can be realized if only toroidal magnetic field exists without vertical electric current in the thin disk.

The rate of energy generation per unit area of one side of the disk is given by (Shakura \& Sunyav 1973, Novikov \& Thorne 1973, Gierliński et al. 1999; Shafee et al.(2008),

$$
Q(R)=-R H \alpha p \frac{d \Omega_{R}}{d R}=-\frac{\dot{M}\left(R^{2} \Omega_{R}-R_{m s}^{2} \Omega_{m s}\right)}{4 \pi R} \cdot \frac{d \Omega_{R}}{d R},
$$

where $\dot{M}$ and $\alpha$ are respectively the mass accretion rate and viscosity parameter, $\Omega_{R}$ and $\Omega_{m s}$ denote respectively the angular velocity at $R$ and inner edge of disk. The radius $R_{m s}$ of the innermost stable circular orbit (ISCO) of accretion disk varies with $a_{*}$ in Pseudo-Kerr potential, Mukhopadhyay (2002) presented that $r_{m s}\left(r_{m s}=R_{m s} / R_{g}\right)$ satisfies the following equation

$$
-3 a_{*}^{4}+14 a_{*}^{3} \sqrt{r_{m s}}+\left(r_{m s}-6\right) r_{m s}^{3}+6 a_{*} r_{m s}^{3 / 2}\left(r_{m s}+2\right)-2 a_{*}^{2} r_{m s}\left(r_{m s}+11\right)=0 .
$$

It is easy to find that ISCO moves to the BH with the increasing $a_{*}$ as shown in Fig.1.Thus the local radiation flux $F(r)$ can be written as:

$$
F(r)=\frac{\dot{M} c^{6}}{8 \pi G^{2} M^{2} r^{4}} g\left(r, r_{m s}, a_{*}\right)
$$

where we have $r=R / R_{g}$, and

$$
\begin{aligned}
g\left(r, r_{m s}, a_{*}\right)= & \left(2 r^{2} \sqrt{r_{m s}}-r^{2} \sqrt{r_{m s}^{3}}-r^{2} a_{*}+2 a_{*} \sqrt{r r_{m s}^{3}}-a_{*}^{2} \sqrt{r_{m s}^{3}}+r_{m s}^{2} \sqrt{r^{3}}-2 r_{m s}^{2} \sqrt{r}\right. \\
& \left.+r_{m s}^{2} a_{*}-2 a_{*} \sqrt{r^{3} r_{m s}}+a_{*}^{2} \sqrt{r^{3}}\right)\left(12 \sqrt{r^{5}} a_{*}-3 r^{4}+16 a_{*}^{2} r-16 a_{*} \sqrt{r^{3}}-7 r^{2} a_{*}^{2}\right. \\
& \left.+2 r^{3}-4 a_{*}^{3} \sqrt{r}\right) /\left[\sqrt{r}\left(\sqrt{r^{3}}-2 \sqrt{r}+a_{*}\right)^{3}\left(\sqrt{r_{m s}^{3}}-2 \sqrt{r_{m s}}+a_{*}\right)\right]
\end{aligned}
$$




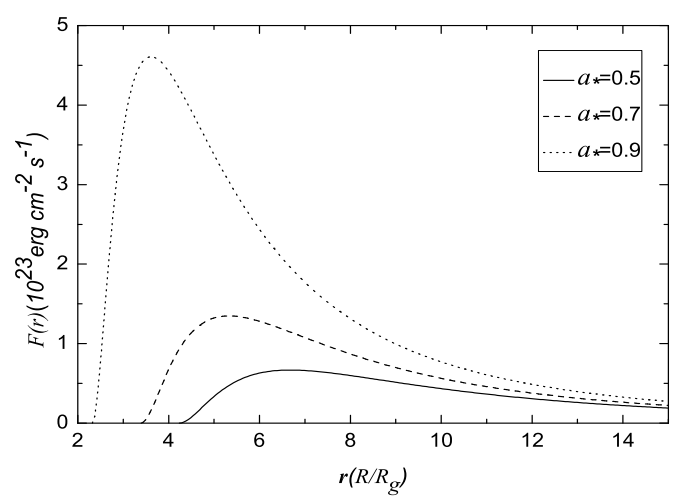

Fig. 1 The curves of local radiation flux $F(r)$ versus $\mathrm{r}$ with the given values of $a_{*}$. ( $M=$ $10 M_{\odot}, \dot{M}=0.1 \dot{M}_{E d d}, \dot{M}_{E d d}$ is the Eddington accretion rate.)

By using equation (20) and (21), we plot the curves of local flux $F(r)$ versus $r$ as shown in Fig.1, and we find that the radiation flux is dominantly produced in the inner disk, and it varies non-monotonically with $r$, attaining its peak value at $r_{d}$ close to ISCO. In addition, as shown in Fig.1, we find that the peak value of $F(r)$ is greater, and the location of $r_{d}$ is closer to ISCO for a greater BH spin.

Inspecting equations (18)-(21), we can find that the radiation flux is directly caused by the angular momentum transport. For magnetized thin accretion disk, numerical simulations have shown that the angular momentum transport is dominated by Maxwell stresses (Hawley et al.1995, Brandenburg et al.1995, Stone et al. 1996), and the magnetic contribution to viscosity parameter $\alpha$ exceeds fluid stresses by an order of magnitude, so that $\alpha \simeq \alpha_{\text {magnetic }}$ (Armitage and Natarajan 1999). So in low mass X-ray binaries the high energy X-ray radiation is generated mainly from the interaction of the plasma with the magnetic field, and the change of the magnetic field could modulate the X-ray flux (Shi 2011). Thereby the oscillations of the toroidal Alfvén wave may lead to the QPOs. Since the strongest influence of the perturbation on luminosity corresponds to the maximum radiation flux $F_{\max }(r=r d)$, the perturbation frequency at $r_{d}$ can be regarded as the LFQPO frequency.

\section{FITTING LFQPOS OF BHXBS}

In this section we intend to fit the LFQPOs of several BHXBs based on the above relation between LFQPO frequency and the perturbation frequency. In addition, the positive correlation between LFQPO frequency and disk flux can be well interpreted based on our model.

We consider a geometrically thin, optically thick accretion disk with corona floating above given by Gierliński et al. (1999). In the inner region of weak magnetic fields, the gas pressure and magnetic pressure can be neglected, and the total pressure is

$$
p \simeq p_{\text {rad }}=\frac{4 \sigma}{3 c} T_{c},
$$

where $\sigma$ is Stefan-Boltzmann constant, and the quantity $T_{c}$ is the temperature of the central disk, which is determined by the equilibrium between the radiation cooling in the vertical direction and the energy generated by the viscous dissipation. We assume that a fraction $q$ of the total energy generated by the viscous process is emitted from the disk, and the remainder is dissipated in the corona. This energy equation is written as (Frank, King \& Raine 2002; Gierliński et al.[1999)

$$
\frac{4 \sigma}{3 \tau} T_{c}^{4}=q F(r) .
$$


Table 1 Fitting LFQPO Frequencies of X-ray BHBs.

\begin{tabular}{cccccc}
\hline source & $\nu_{Q P O}^{a}$ & $m_{B H}^{b}$ & $a_{*}^{b}$ & $\beta=p_{\text {mag }} / p$ & $\dot{m}$ \\
\hline GRO J1665-40 & $0.1-28$ & 6.2 & 0.7 & & $0.00086-0.24$ \\
XTE J1550-564 & $0.1-10$ & 9.1 & 0.34 & 0.01 & $0.0023-0.23$ \\
GRS 1915+105 & $0.001-10$ & 15.0 & 0.975 & & $0.00007-0.07$ \\
4U 1543-47 & 7 & 9.4 & 0.8 & & 0.073 \\
\hline
\end{tabular}

Notes: ${ }^{a}$ Remillard \& McClintock (2006); ${ }^{b}$ Narayan \& McClintock (2011)

The parameter $\tau$ in equation (24) is the opacity for electron scattering, and it reads

$$
\tau \cong \rho H \sigma_{T} / m_{p},
$$

where $\sigma_{T}$ and $m_{p}$ denote the Thomson cross-section and proton mass, respectively. Incorporating equation (11) with equations (23)-(25), we obtain

$$
H=\frac{\sigma_{q T}}{m_{p} c\left(\Omega_{k}\right)^{2}} F(r) .
$$

The Eddington accretion rate $\dot{M}_{E d d}$ can be written as

$$
\dot{M}_{E d d}=2 \pi R_{m s} m_{p} c / \eta \sigma_{T},
$$

where $\eta$ is the accretion efficiency, whose value is 0.1 for black hole. Substituting equations (6), (21) and (27) into equation (26), we have

$$
H=3.7 \times 10^{5} m_{B H} \dot{m} q \frac{r_{m s}\left(\sqrt{r^{3}}-2 \sqrt{r}+a_{*}\right)^{2}}{\left(2 a_{*} \sqrt{r}-r^{2}-a_{*}^{2}\right)^{2}} g\left(r, r_{m s}, a_{*}\right),
$$

where we define $m_{B H} \equiv M / M_{\odot}$ and $\dot{m} \equiv \dot{M} / \dot{M}_{E d d}$.

Combining equations (6), (18) and (28), we have the perturbation frequency as a function of disk radius $r$, and we derive the expression for LFQPO frequency by setting $r=r_{d}$ as follows,

$$
\nu_{Q P O}=\frac{\omega}{2 \pi}=5.1 \times 10^{5} \frac{\sqrt{\beta} \dot{m} q}{m_{B H}} \frac{r_{m s}\left(\sqrt{r_{d}^{3}}-2 \sqrt{r_{d}}+a_{*}\right)}{r_{d}^{3}\left(2 a_{*} \sqrt{r_{d}}-r_{d}^{2}-a_{*}^{2}\right)} g\left(r_{d}, r_{m s}, a_{*}\right) .
$$

From equation (29) we find that the LFQPO frequency is proportional to accretion rate $\dot{m}$, but it is inversely proportional to the $\mathrm{BH}$ mass $m$. Based on the above results we can fit some LFQPO frequencies of several BHXBs with the given $\mathrm{BH}$ mass by adjusting the mass accretion rate and the intensity of magnetic field $\beta$, and the main results are summarized as follows.

First, the theoretical values of the LFQPO frequencies are in accordance with the observed ones with appropriate values of $\dot{m}$ and $\beta$. We assume that the dissipation in corona is neglected $(q=1)$, and $\beta=$ $p_{\text {mag }} / p=0.01$, and the fitting results are listed in Table 1 .

Second, the observed strong positive correlation between the LFQPO frequencies and the disk flux can be interpreted very well based on our model.

We assume that the disk locally emits a blackbody spectrum, of which a fraction $1-p_{s c}$ is scattered in the corona, and the disk luminosity is (Gierliński et al. 1999)

$$
L_{s}=p_{s c} q \eta \dot{M} c^{2}=\frac{2 \pi D^{2} F_{s}}{\cos i},
$$

where $i$ and $D$ are the inclination of the disk and the source distance to the observer, respectively. The quantity $\left(1-p_{s c}\right)$ is the fraction of the disk emission which is not scattered by the corona, and $F_{s}$ is the observed disk flux. Combining equations (29) and (30), we obtain the relation between the LFQPO frequency and the observed disk flux $F_{s}$ as follows.

$$
\nu_{Q P O}=2.5 \times 10^{-33} \frac{\sqrt{\beta} F_{s} D^{2}}{m_{B H}^{2} p_{s c} \cos i} \frac{r_{m s}\left(\sqrt{r_{d}^{3}}-2 \sqrt{r_{d}}+a_{*}\right)}{r_{d}^{3}\left(2 a_{*} \sqrt{r_{d}}-r_{d}^{2}-a_{*}^{2}\right)} g\left(r_{d}, r_{m s}, a_{*}\right) .
$$


Table 2 LFQPO Frequencies Fitting Parameters of XTE J1550-564 and GRO J1655-40.

\begin{tabular}{cccccccccc}
\hline source & Input & & & & \multicolumn{3}{c}{ Output } & Fitting parameter & $\chi^{2}($ dof $)$ \\
& $m_{B H}$ & $a_{*}$ & $D(k p c)$ & $i$ & $p_{s c}$ & $r_{m s}$ & $r_{d}$ & $\beta=p_{m a g} / p$ & \\
\hline XTE J1550-564 & 9.1 & 0.34 & 4.38 & $75^{0}$ & 0.15 & 4.83 & 7.64 & $0.0105 \pm 0.0003$ & $44.9(32)$ \\
GRO J1665-40 & 6.3 & 0.7 & 3.2 & $70^{0}$ & 0.1 & 3.39 & 5.33 & $0.0159 \pm 0.0005$ & $61.2(37)$
\end{tabular}

Notes:The values of input parameters $\left(m_{B H}, a_{*}, D, i\right)$ are adopted from Narayan \& McClintock (2011). The quantities of $r_{m s}$ and $r_{d}$ are computed by using equations (20) and (21).

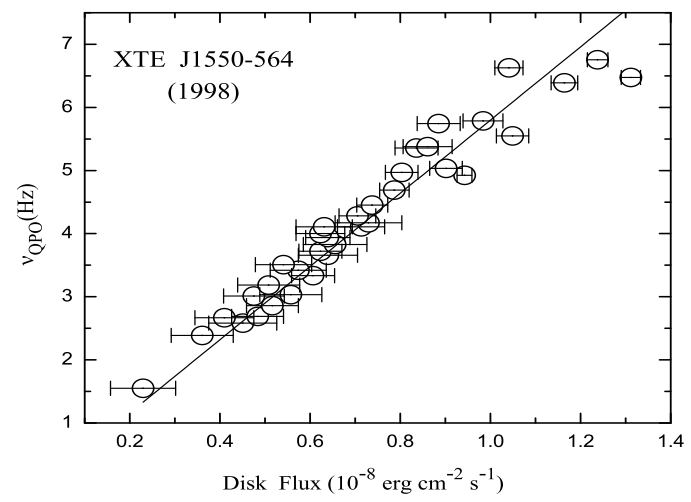

Fig. 2 Fitting the relation between the disk flux and LFQPO frequency for XTE J1550-564. The observation data are taken from Fig. 2 Of SobcZak et al. (2000), while the flux errors are corrected based on McClintock et al. (2009).

From equation (31) we find that LFQPO frequency has a positive correlation with the observed disk flux. For XTE J1550-564 (outburst in 1998) and GRO J1655-40 (outburst in early 2005). The values of $p_{s c}$ are about 15\% (Sobczak et al. 2000) and 10\% (Shaposhnikov et al.2007), respectively, and they keep constant in hard state. Therefore their disk fluxes have a linear relation with LFQPO frequencies.

For a given source, the values of $r_{m s}$ and $r_{d}$ can be obtained by resolving equations (20) and (21), and the values of the parameters, such as $m_{B H}, a_{*}, D, i$, and $p_{s c}$ )are available in the literatures. Substituting all the parameters into the equation (31), we can derive the LFQPO frequency corresponding to disk flux $F_{s}$ and the magnetic field parameter $\beta$ as follows,

$$
\nu_{Q P O}=f(\beta) F_{s},
$$

where $f(\beta)$ is the function of $\beta$. The measurement values of LFQPO frequency and disk flux are $\nu_{i}$ and $F_{i}\left(i=1,2, \cdots, N\right.$, the number of the observation data), of which the errors are $\epsilon_{\nu i}$ and $\epsilon_{F i}$. We adopt the Nukers' estimate (Tremaine et al.2002) based on the following minimizing

$$
\chi^{2} \equiv \sum_{i=1}^{N} \frac{\left(\nu_{i}-f(\beta) F_{i}\right)^{2}}{\epsilon_{\nu i}^{2}+f^{2}(\beta) \epsilon_{F i}^{2}} .
$$

Using the Nukers' estimate, we can derive the best fitting parameter $\beta$ for XTE J1550-564 and GRO J1655-40, which are presented in Table 2, and the values of reduced $\chi^{2}$ per degree of freedom are less than 2 for both sources, indicating that LFQPO frequency has approximate linear relation with disk flux as shown in Figs. 2 and 3 for XTE J1550-564 and GRO J1655-40, respectively. 


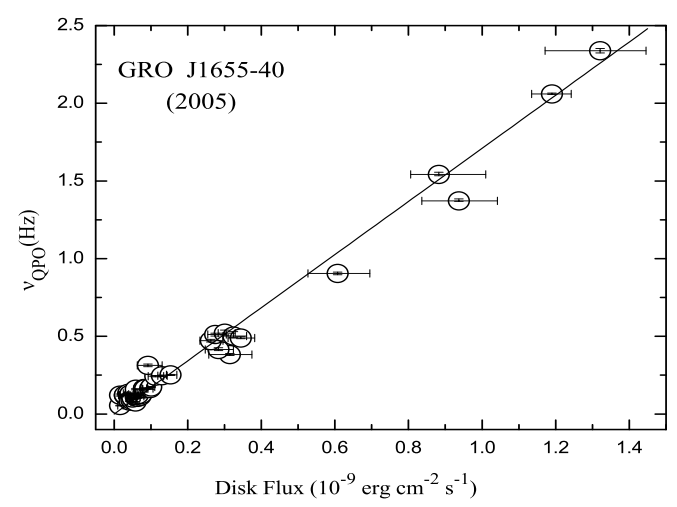

Fig. 3 Fitting the relation between the disk flux and LFQPO frequency for GRO J1655-40. $\left(m_{B H}=6.2, \beta=0.6, D=3.2 \mathrm{kpc}, i=70^{0}\right.$. The observation data are taken from Fig. 15 of Shaposhnikov et al. (2007).

\section{DISCUSSION}

In this paper, we propose that the LFQPOs in BHXBs can be interpreted by invoking the toroidal Alfvén wave oscillations located at the disk radius with the maximal radiation flux. It turns out that the LFQPO frequencies of several BHXBs can be well fitted based on our model. In addition, the positive correlation between the LFQPO frequencies and the disk flux of XTE J1550-564 and GRO J1655-40 are also well fitted. According to the argument given in sections 2 and 3, we find that the positive correlation between LFQPO frequency and the disk flux can be understood, because both the perturbation frequency of the toroidal Alfvén wave and LFQPO frequency increase with the increasing accretion rate. It is noted that this correlation only holds in hard stats, when the disk fraction keeps constant. Thus our model provides an explanation for the fact that LFQPOs are primarily observed in the power law part of spectra of BHXBs (Zycki \& Sobolewska2005).

In this paper, we adopt a thin disk with some corona floating above, which is different from the corona interior to a truncated disk as given by Done, Gierlinski \& Kubota (2007). The truncated disk is not compatible with our model because it is difficult to determine the location of maximal radiation flux. If Alfvén wave of truncated disk is used to interpret QPOs, a certain appropriate location of Alfvén wave propagating must be found out, e.g. Shi (2011) used Alfvén wave oscillation at transition radius to explain HFQPOs of low mass X-ray binaries.

Although LFQPOs are interpreted successfully by invoking perturbed Alfvén wave oscillation, it seems difficult to explain high Frequency QPOs (HFQPOs) of BHXBs. This could imply that the physical origins of LFQPOs and HFQPOs are different. HFQPOs of BHXBs have been successfully explained by the magnetic hot spots model (Wang, et al. 2003, 2005) and resonance mode (Abramowicz and Kluzniak 2001, Huang, et al. 2010), and the HFQPO frequencies are sensitive to BH mass and spin, while they seems not directly related to the accretion rate and disk flux. It is noted that HFQPOs may be triggered by the instability of accretion disk oscillation (Tagger et al. 2006, 2001). We also notice that the instability occurs in the toroidal Alfvén wave oscillation, and the oscillation frequency is comparable to the HFQPO frequency, provided that the accretion rate and the magnetic field are great enough. This result motivates us to explore the relation of HFQPOs and the instability of Alfvén wave oscillation in our future work.

Acknowledgements This work is supported by the NSFC (grants 11173011, 11143001, 11103003 and 11045004), the National Basic Research Program of China (2009CB824800) and the Fundamental Research Funds for the Central Universities (HUST: 2011TS159). 


\section{References}

Abramowicz, M. A., \& Kluźniak, W. 2001, A\&A, 374, L19

Armitage, P. J., \& Natarajan, P. 1999, ApJ, 523, L7

Brandenburg, A., Nordlund, A., Stein, R. F., \& Torkelsson, U. 1995, ApJ, 446, 741

Cabanac, C., Henri, G., Petrucci, P. O., Malzac, J., Ferrera, J., \& Belloni, T. M. 2010, MNRAS, 404, 738

Caunt, S. E., \& Tagger, M. 2001, A\&A, 307, 1695

Done, C., Gierlinski, M., \& Kubota, A. 2007, Astron. Astrophys. Review, 15, 1

Frank, J., King, A., \& Raine, D. J. 2002, Accretion Power in Astrophysics: Third Edition, ed. Frank, J., King, A., \& Raine, D. J.

Gierliński, M., Zdziarski, A. A., \& Poutanen, J., et al. 1999, MNRAS, 309,496

Hawley, J. F., Gammie, C. F., \& Balbus, S. A. 1995, ApJ, 440, 742

Heil, L. M., Vaughan, S., \& Uttley, P. 2011, MNRAS, 411, L66

Huang, C. Y., Gan, Z. M., \& Wang, J. Z., et al. 2010, MNRAS, 403, 1978

Ingram, A., \& Done, C. 2012, MNRAS, 419, 2369

Ingram, A., Done, C. \& Fragile, P. C. 2009, MNRAS, 392, L101

Kato, S. J. 2008, PASJ, 60, 889

Koide, S., Shibata, K., Kudoh, T., \& Meier, D. L. 2002, Science, 295, 1688

Li, L. X. 2002, ApJ, 567, 463

Markwardt, C. B., Swank, J. H., \& Taam, R. E. 1999, ApJ, 513, L37

McClintock, J. E., Remillard, R. A., \& Rupen, M. P., et al. 2009, ApJ, 698, 1398

McClintock, J. E., \& Remillard, R. A. 2006, in Lewin, van der Klis, eds, Compact Stellar X-ray Sources. Cambridge Univ. press,Cambridge, p.157

Muno, M. P., Remillard, R. A., \& Morgan, E. H. 2001, ApJ, 556, 515

Mukhopadhyay, B. 2002, ApJ, 581, 427

Mukhopadhyay, B. 2003, ApJ, 586, 1268

Narayan, R., \& McClintock, J. E. 2011, preprint arXiv:1112.0569)

Novikov, I. D., \& Thorne, K. S. 1973, In: B,De Witts,C.De Witts,eds.,Black Holes,New York:Gordon and Breach, p.343

O’Neill, S. M., Reynolds, C. S., \& Miller, M. C., et al. 2011, preprint (arXiv:1009.1882)

Remillard, R. A., \& McClintock, J. E. 2006, ARA\&A, 44, 49

Revnivtsev, M. G., Trudolyubov, S. P., \& Borozdin, K. N. 2000, MNRAS, 312, 151

Schnittman, J. D., Homan, J., \& Miller, J. M. 2006, ApJ, 642, 420

Shafee, R., Narayan, R., \& Mcclintock, J. E. 2008, ApJ, 676, 549

Shakura, N. I., \& Sunyaev, R. A. 1973, A\&A, 24, 337

Shaposhnikov, N., Swank, J., \& Shrader, C. R., et al. 2007, ApJ, 655, 434

Shi, C. S. 2011, RAA, 11, 1327

Shi, C. S., \& Li, X. D. 2010, ApJ, 714, 1227

Sobczak, G. J., McClintock, J. E., \& Orosz, J. A., et al. 2000, ApJ, 531, 537

Stella, L., \& Vietri, M. 1998, ApJ, 492, L59

Stone, J. M., Hawley, J. F., Gammie, C. F., \& Balbus, S. A. 1996, ApJ, 463, 656

Tagger, M., \& Pellat, R. 1999, A\&A, 349, 1003

Tagger, M., \& Varnière, P. 2006, ApJ, 652, 1457

Timarchuk, L., \& Osherovich, V. 2000, ApJ, 542, L111

Tremaine, S., Gebhardt, K., \& Bender, R. 2002, ApJ, 574, 740

Wang, D. X., Ma, R. Y., \& Lei, W. H., et al. 2003, ApJ, 595, 109

Wang, D. X., Ye, Y. C., \& Yao, G. Z., et al. 2005, MNRAS, 359, 36 
Zycki, P. T., \& Sobolewska, M. A. 2005, MNRAS, 364, 891

Zhang, C. 2004, A\&A, 423, 401 